\title{
PUBBLICAZIONI RECENTI
}

Rasbe. - Mathematische Mittheilungen -. Zweites Heft. Zïrich. 1268.

(Coniene le seguenti memorie - Sull'introdursi dell'imnaginario nella valutazione degli integrali definiti doppi a limiti reali di significato geometrico - . Alcune applicazioni della serie di Stirling generalizzata - Sulle funzioni Bernoulliane ed Euleriane - . Sulla integrazione della equazione a derivate parziali $s^{2}-r t=f(p, q)$.)

Sazmon. - On the degree of the surface reciprocal to a given one - Transactions of Irish Academy. Vol. 23.

Bools. - On the Application of the Theory of Probabilities to the Question of the Combination of Testimonies or Judgments - Transactions of the $\mathbf{R}$. Society of Edinburgh. Vol. 21.

Beulavitis. - Sposizione elementare della teorica dei determinanti. Memorie dell'Istituto Veneto. Vol. 7.

Fuaurr. - Sulla vera nozione delle quantità negative risultanti dalla risoluzione dei problemi.

DeL-Grosso. - Sulle funzioni generatrici di alcune rimarchevoli serie trascendenti.

Batraglins. - Sulla dipendenza scambievole delle figure. - Sulla partizione dei numeri.

Pabula. - Ricerche sulle superficie curve.

Gasparis. - Sopra una equazione di grande importanza nella teorica dei movimenti dei pianeti. Memorie della R. Accademia di Napoli. Vol. 2. 1857.

Cayley. - On the Cubic Transformation of an Elliptic Function - Philosophical Magazine. May. 1858.

Kronecker. - Ueber Gleichungen des Siebenten Grades. Monatsbericht der Academie zu Berlin. April. 1858.

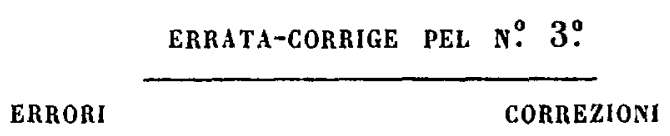

pag. 129 lin. 15.

$x_{1}^{2} \frac{\mathrm{d} \varphi}{\mathrm{d} x_{\mathrm{I}}}+x_{2}^{2} \frac{\mathrm{d} \varphi}{\mathrm{d} x_{2}}+\ldots+x_{n}^{2} \frac{\mathrm{d} \varphi}{\mathrm{d} x_{n}}+n a_{1} \frac{\mathrm{d} \varphi}{\mathrm{d} a_{o}}, y\left(x_{1}^{2} \frac{\mathrm{d} \varphi}{\mathrm{d} x_{\mathrm{s}}}+x_{2}^{2} \frac{\mathrm{d} \varphi}{\mathrm{d} x_{2}}+\ldots+x_{n}^{2} \frac{\mathrm{d} \varphi}{\mathrm{d} x_{n}}+n a_{1} \frac{\mathrm{d} \varphi}{\mathrm{d} a_{0}}\right)$

pag. 131, lin. 3.

$$
2 \alpha_{n-1, n}=(n-2 t) \theta+m \quad 2 \alpha_{n-1, n}+\alpha_{0, \mathrm{1}}+\ldots+\alpha_{0, n}=(n-2 t) \theta+m
$$

